\title{
CONTENTION WINDOW AND RESIDUAL BATTERY AWARE MULTIPATH ROUTING SCHEMES IN MOBILE AD-HOC NETWORKS
}

\author{
Valmik Tilwari ${ }^{1}$, MHD Nour Hindia ${ }^{1}$, Kaharudin Dimyati $^{1 *}$, Faizan Qamar ${ }^{1}$, \\ Mohamad Sofian Abu Talip ${ }^{1}$ \\ ${ }^{1}$ Department of Electrical Engineering, Faculty of Engineering, University of Malaya, 50603, Kuala \\ Lumpur, Malaysia
}

(Received: November 2018 / Revised: January 2019 / Accepted: August 2019)

\begin{abstract}
In mobile ad hoc networks, limited energy resources and traffic congestion at the nodes are crucial issues due to the nodes being battery operated and flooding the network with packets, respectively. These issues degrade network routing performance in terms of quality of service. In this study, we proposed a contention window and residual battery-aware multipath routing scheme to enhance network performance. Our proposed routing scheme has successfully diverted the traffic load from a low energy node to a high energy node while also controlling congestion among intermediate nodes. A multi-criteria decision-making technique was also used for the selection criteria of an intermediate node in the optimal path, based on the mobility and window size contention of nodes. Eventually, the contention window and residual battery-aware multipath routing scheme has enhanced throughput, attenuated the packet loss ratio, and reduced the energy consumption in comparison to a conventional multipath optimized link state routing protocol routing scheme.
\end{abstract}

Keywords: CRAM; Mobile ad hoc networks; Multi-criteria decision-making; Multipath optimized link state routing protocol; Quality of service

\section{INTRODUCTION}

Rapid wireless technology development escalates upon the demands of its users. In 2019, people want everything to be controlled by their fingertips. Among the notable applications used in wireless technologies are mobile ad hoc networks (MANETs; Jabbar et al., 2017), device-todevice communication (Mumtaz et al., 2014), the Internet of things (Atzori et al., 2010), cognitive radio (Badoi et al., 2011), and heterogeneous networks (Peng et al., 2015). These technologies offer the biggest potential for reliable end user communication. Among them, MANETs provide prime solutions for user demand with self-organized and infrastructureless networks. In MANETs, user nodes collaborate with each other and acts as routers with end users building ad hoc networks for communication. To determine and maintain the best path for transferred data between source and destination nodes, optimal routing protocols for better quality of service (QoS) in the network need to be identified.

MANET routing protocols can be classified into three types, depending on their functionalities of instant reactive, proactive, and hybrid routing protocols. In reactive routing protocols, such as destination-sequenced distance-vectors (Hamid et al., 2015), and optimized link state routing protocol (OLSR; Yi \& Parrein, 2017), source nodes initiate a route discovery process to

\footnotetext{
"Corresponding author's email: kaharudin2k18@gmail.com, Tel. +60-0379677641

Permalink/DOI: https://dx.doi.org/10.14716/ijtech.v10i7.3268
} 
transmit the data. By contrast, in proactive routing protocol (OLSR; Yi \& Parrein, 2017), source nodes initiate a route discovery process to transmit the data. By contrast, in proactive routing protocols, such as dynamic source routing (Hui et al., 2016) and ad hoc on-demand distance vectors (Kabir, et al., 2015), every node always has network topology information in the form of table, owing to periodic transfer messages in the network. Whenever a source node needs to transmit packets, it will take routing information from the table to establish a network path. In hybrid routing protocols, such as zone routing protocol (Lin et al., 2017) and secure link state routing protocol (Sarkar et al., 2016), the routing decisions are made based on the geographical location of nodes to attain higher efficiency and scalability. However, if a destination node is in a given geographical area, it will use table-based routing, while destination nodes outside the geographical location use on-demand routing protocols.

Table-based routing protocols have one major drawback: every node in the network must exchange "HELLO" and "topology control" messages continuously with neighboring nodes. Such messaging increases the load burden of a network (i.e., traffic overhead). By contrast, ondemand routing protocol establishes routes only if a source node needs to transfer data, reducing the resultant load on the network. While research has been conducted on single path routing under a OLSR on-demand routing protocol (Sun et al., 2016), this protocol causes rapid energy depletion at the node due to high traffic congestion on a single node. That congestion degrades network performance and increases the possibility of link failure in the network, affecting packet loss and end-to-end delay (Li et al., 2017).

The multipath optimized link state routing protocol (MP-OLSR; Yi et al., 2011) resolves such issues by selecting multiple routes using multipath Dijkstra algorithms to establish connections between source and destination nodes. Moreover, the route selection process provides efficient communication and load balance among nodes by distributing packets to multiple paths. To solve the continuous exchange message flooding problem under the MP-OSLR protocol, the multipoint relay (MPR) concept has been introduced. MPR nodes are relay nodes that have at least two nexthop neighbor nodes. To mitigate network overhead, a source node only sends data packets to MPRs nodes. Energy efficient nodes are selected as the MPRs, so more reliable and robust route can be established by prolongs the lifetime of the route and network. However, the MP-OLSR routing scheme still faces challenges during the route selection process, due to rapid node depletion and traffic congestion on available paths. Based on these circumstances, this paper will focus on the selection of optimal routes from source to destination nodes in MANETs. We shall consider the status of the intermediate node during optimal route selection in terms of residual battery $(\mathrm{RB})$ and contention window $(\mathrm{CW})$ to improve the QoS. Moreover, the multi-criteria decision-making (MCDM) method will be used to determine the criteria of suitable nodes within an optimal route. Overall, the proposed routing scheme will be compared with the existing MPOLSR routing scheme, the results will be expressed in the terms of the throughput, packets loss ratio, and energy consumption with various node speed.

The rest of this paper is organized as follows: section 2 illustrates the related works; section 3 describes the system model for optimal route selection; section 4 presents the simulation model results and discusses them further; and section 5 draws the conclusion.

\section{RELATED WORK}

Several studies and innovations have been conducted on using the MP-OLSR routing scheme in MANETs, covering subjects such as energy efficiency, bandwidth scalability, and the mobility of node constraints (Sarobin \& Thomas, 2016; Tilwari et al., 2019). This paper has reviewed and illustrated some of the recent literature in this section. (Sarkar \& Datta, 2017) presented a route selection technique that introduced trust-based mobility aware routing to estimate the mobility 
factor value using the pause time, speed, and moving angular direction of mobile nodes for static and dynamic scenarios. Mobility aware routing selects nodes with high threshold values of mobility factor between a source and destination to improve network stability. Rajeswari and Neduncheliyan (2017) proposed a smooth mobility and link reliability-based OLSR routing scheme to address the chance of link failure in MANETs. A semi-Markov smooth and complexity restricted mobility model was used to provide sufficient smoothness and low complexity for reliability and efficient MPR selection, providing longer lifetimes for MPRs and networks.

Villasenor-Gonzalez et al. (2005) presented a heterogeneous OLSR scheme to resolve the scalability problem of reactive routing schemes in ad hoc networks. Their scheme offered communication with multiple wireless interfaces, such as multiple radio interfaces, while reducing routing overhead. Boushaba et al. (2014) built on that discovery to introduce an extension version of MP-OLSR for heterogeneous multipath networks called fuzzy-based QoS MP-OLSR. In MANETs, video transmission is challenging owing to limited resource availability and network instability. The protocol established routes in two stages: QoS (throughput, delay, and signal-to-interference-plus-noise ratio) and weight (based on the link cost function). A weighted round-robin technique was used to schedule data packets, enhancing video streaming quality considerably in terms of QoS.

Meanwhile, Wang et al. (2014) presented a table-based source routing protocol to facilitate opportunistic data forwarding in MANETs. In that protocol, each node possessed full information about the network topology by periodically exchanging messages and link cost information (based on the number of nodes in the path) with its neighbors. The protocol used less routing overhead compared to ad hoc on-demand distance vector, OLSR, and dynamic source routing schemes with better data transportation capability. Fatima and Najib (2012) presented a route selection technique based on node mobility and modified MPR selection criteria. Their paper enhance the network performance, as existing MP-OLSR schemes do not consider mobility and MPR selection criteria when selecting optimal routes. Joshi and Rege (2012) introduced a modified OLSR scheme that based its routing on the energy awareness and drain rate of mobile nodes for reliable routing and energy conservation. It provided load balancing among nodes and increased the number of live nodes. Another approach taken by (Yi et al., 2011) proposed a MPOLSR routing scheme based on the multipath Dijkstra algorithm. The scheme discovers routes based on the link cost function of the path, which is determined by network stability and wireless transmission instability. That study introduced two new concepts, namely route recovery and loop detection, which would improve network performance, especially in high-mobility and heavyloaded network scenarios (Yi et al., 2011). Mobile node battery exhaustion is a significant challenge for MANETs, as the battery is draining, the network will be "dead". To overcome such exhaustion, a study by Jabbar et al. (2014) presented an mobility and battery aware OLSR scheme to improve network lifetimes with lower energy costs per packet. The scheme's energy efficient routing established routes and prolonged network lifetimes to reduce link failure owing to node battery exhaustion. As can be seen from above-mentioned literature, from the authors best knowledge there is no research that combined contention window and residual energy parameters in the selection of optimal route. Owing to this, the proposed method is utilizing these parameters to boost the network performance and QoS.

\section{SYSTEM MODEL}

A MANET is represented by the graph $G(N, L)$, where $N$ denotes the number of nodes and $L$ denotes the links between nodes. Let $s$ define the source node, $d$ define the destination node, and let the link between them be defined by $(s, d)$. The transmission rate matrix of the whole network is defined by $r(t)$, which includes the topology, traffic condition, and link quality of the 
network. The rate of the transmission matrix between source $s$ and destination $d$ is represented by $\mu_{(s, d)}(t)$, which varies continuously due to the mobility of nodes while the network topology changes randomly. The source node starts its transmission of data packets through multiple paths with intermediate nodes. The rate of data transmission on the link $(s, d)$ with intermediate node $c$ defined by $\mu_{(s, d)}^{c}(t)$ i.e. $\mu_{(s, d)}(t) \geq \mu_{(s, d)}^{c}(t) \in r(t)$. The source node initiates the route discovery process using multipath Dijkstra algorithms to gather information about RB and CW of the intermediate node to select the optimal route between source and destination. The energy and $\mathrm{CW}$ model are described in sections 3.1 and 3.2, respectively.

\subsection{Energy Model}

In a CRAM routing scheme, the RB on a node plays an important role in selecting an optimal path between the source and destination nodes due to the link breakage problem. The RB is defined as the amount of battery remaining on a node from its maximum value, which is gained from the linear battery model (Tremblay et al., 2007). The intermediate nodes are selected based on their RB status. If RB value of a node is high, the node will receive higher priority for the

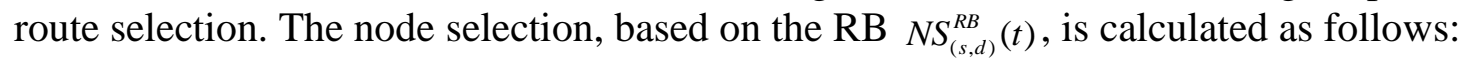

$$
N S_{(s, d)}^{R B}(t)=\frac{R B_{c}(t)}{R B_{\max }(t) \times D R_{c}(t)},
$$

where $R B_{c}(t)$ is defined as the RB on an intermediate node $c, R B_{\max }(t)$ is the maximum battery of all nodes, and $D R_{c}(t)$ refers to the drain rate of $c$. The drain rate is calculated as follows:

$$
D R_{c}(t)=\frac{E_{t o t a l} \times V}{T}
$$

where $E_{\text {total }}$ is the total energy resource for all nodes, $V$ defined as the supply voltage on a node, and $T$ is tracking time of an intermediate node $c$.

\subsection{CW Model}

The CW is defined as the window size of a node that allows the transferring of data packets. The CW size is the amount of time that the nodes are compelled to wait after a collision occurrence before accessing channels for subsequent data transmission. $\mathrm{CW}$ adjusts the size of packets needed for transmission on the link to avoid packet collision and improve congestion control. The CW size of intermediate nodes is a critical factor in the construction of optimal routes; as a result, only intermediate nodes with lower CW sizes are selected during the building of optimal routes. It is calculated based on the size of the node's queue length at the time of the route discovery process. The $\mathrm{CW}$ receives an acknowledgment message from the destination node, which includes information about the queue length value of the intermediate nodes. If the queue size at a node is high, its $\mathrm{CW}$ size will decrease and vice versa. The $\mathrm{CW}$ size also controls the data rate of transmission on the link. The CW size is calculated as follows:

$$
N S_{(s, d)}^{C W}(t)=\frac{e^{\left\{Q L_{\text {avg }}^{c}(t)-Q L_{\text {new }}^{c}(t)\right\}}}{\mu_{(s, d)}^{c}(t)},
$$

where $Q L_{\text {avg }}^{c}(t)$ and $Q L_{\text {new }}^{c}(t)$ are defined as the respective average and current queue lengths, measured in bytes, at node $c$. If the data transmission rate and queue length size are high at a node, the $\mathrm{CW}$ size will decrease to provide a higher chance for accessing the channel.

\subsection{MCDM}

The node selection is made based on the energy and CW models. MCDM uses these values to make a decision, based on the node statuses, on the establishment of an optimal path between 
source and destination nodes. These values are monitored continuously when the route is established, but once the status of intermediate node falls below the threshold level, the MCDM automatically switches to another node with a higher RB value and lower CW. The MCDM decision is calculated as follows:

$$
M C D M=\left\{W_{R B} \times N S_{(s, d)}^{R B}(t)+W_{C W} \times N S_{(s, d)}^{C W}(t)\right\},
$$

where $W_{R B}$ and $W_{C W}$ are the weights assigned by the MCDM method based on the amount of RB and the size of CW on each node, respectively.

\subsection{Simulation Setup}

Simulations were completed with MATLAB software version 2018a. They were carried out on a network area size of $1000 \times 1000 \mathrm{~m}^{2}$ with 49 nodes distributed randomly throughout. The transmission range among the nodes was $270 \mathrm{~m}$. In addition, two types of intermediate nodes were considered according to their $\mathrm{RB}$ and $\mathrm{CW}$ size. The constant bit rate traffic has been generated 20 with packet size 512 bps. To access the channel for ad hoc networks, PHY 802.11b, MAC layer has been used to avoid possible collisions with the channel frequency of $2.4 \mathrm{GHz}$. Table 1 shows other simulation parameters.

Table 1 Simulation parameters value

\begin{tabular}{lc}
\hline \multicolumn{1}{c}{ Parameters } & Values \\
\hline Routing schemes & CRAM and MP-OLSR \\
Simulation area & $1000 \times 1000 \mathrm{~m}^{2}$ \\
Simulation time & 600 seconds \\
Number of nodes & 49 \\
Constant Bit Rate & 20 \\
Traffic packets size & 512 bytes \\
Transmitted Signal Power & $\mathrm{P}_{\mathrm{t}=}=31.623 \mathrm{~mW}$ \\
Transmission range & 270 meters \\
Transport protocol & UDP \\
Network protocol & IPv4 \\
MAC protocol & IEEE 802.11 \\
Physical layer model & PHY 802.11b \\
Wireless channel frequency & $2.4 \mathrm{GHz}$ \\
\hline
\end{tabular}

\subsection{Performance Metric}

The main purpose of the CRAM routing scheme is to enhance the network performance in terms of load balancing and congestion control for the nodes. Therefore, we considered the following performance evaluation metrics.

\subsubsection{Throughput}

Throughput can be defined as the average data rate that has been successfully transmitted over a specific link, measured in bits per second (bps). It is calculated as follows:

$$
\text { Throughput }=\frac{P_{r e c} \times 8}{T} \text {, }
$$

where $P_{\text {recieve }}$ is the total number of bytes received and $T$ is the time duration between the first and last bytes received at the destination node.

\subsubsection{Packet loss ratio (PLR)}

PLR is defined as the packets lost during data transmission to the destination node. It is calculated as the percentage ratio between the number of packets lost and number of packets received. 


$$
P L R=\frac{\left(P_{\text {send }}-P_{\text {recieve }}\right) \times 100}{P_{\text {send }}},
$$

where $P_{\text {send }}$ and $P_{\text {recieve }}$ are the number of packets sent by the source node and received by the destination node, respectively.

\subsubsection{Energy Consumption $\left(E_{\text {cons }}\right)$}

Energy consumption is described as the average battery consumption of the intermediate nodes during the data packet transmission between the source and destination nodes. It is calculated as follows:

$$
E_{\text {cons }}(t)=\frac{1}{n} \sum_{c=1}^{n} E_{\text {total }}(t)
$$

where $E_{\text {total }}(t)$ refers to the total energy consumption of node $c$ and $n$ is the total number of intermediate nodes used during the data transmission.

\section{RESULTS AND DISCUSSION}

Extensive simulations were conducted with MATLAB 2018a simulator to evaluate the performance of the CRAM routing scheme. The scheme was subsequently compared with a MPOLSR routing scheme while using different node speed scenarios. The user datagram protocol was used as the transport layer protocol as opposed to the transmission control protocol, providing a simpler transmission model. The random waypoint mobility model was used as the mobility standard, enabling node speed variations from $10 \mathrm{~m} / \mathrm{s}$ to $60 \mathrm{~m} / \mathrm{s}$. The results are illustrated in terms of throughput, PRL, and energy consumption in sections 4.1, 4.2, and 4.3, respectively.

\subsection{Throughput}

As shown in Figure 1, our scheme outperformed the conventional MP-OLSR by controlling the rate of data transmission through the $\mathrm{CW}$ method. The data transmission depended upon the size of the CW available on the established path. The CRAM routing decision was made based on residual energy and CW size of the network nodes, outperforming the other scheme by selecting intermediates nodes with higher residual energy and lower CW size. The throughput performance of the CRAM scheme was around $49.2 \mathrm{kbps}$ at the minimum speed of $10 \mathrm{~m} / \mathrm{s}$ and as high as 43.9 $\mathrm{kbps}$ at the maximum speed of $60 \mathrm{~m} / \mathrm{s}$. The intuitional comparison showed that the CRAM scheme performed $12 \%$ better at throughput than a traditional MP-OLSR routing scheme.

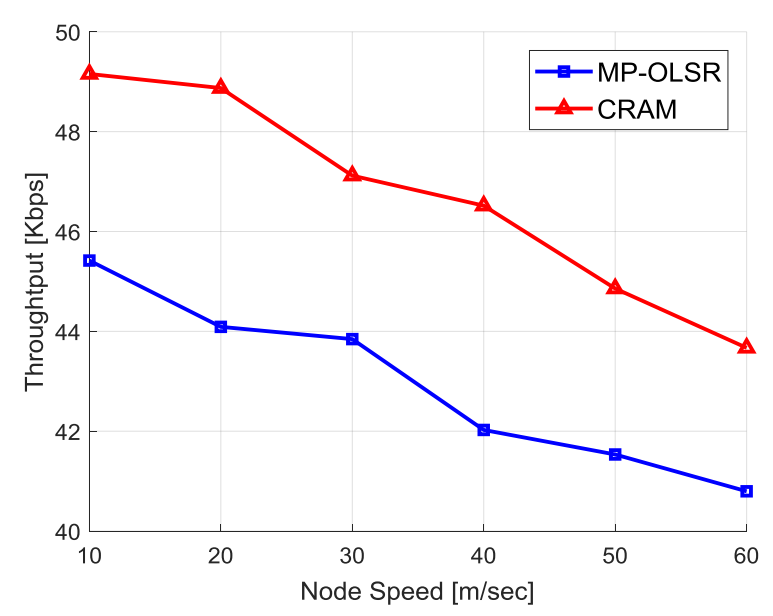

Figure 1 Throughput with different node speeds 


\subsection{PLR}

It can be seen from the Figure 2 that the PLR values were lower for paths established by the CRAM routing scheme. This is due to the nodes avoiding paths with lower amounts of battery remaining to reduce the chance of frequent link failure. Moreover, the CRAM scheme utilized the CW method to reduce PLR value, sending smaller numbers of packets where loads were high on the link. In other words, the CRAM routing scheme forwarded data packets toward lower queue length intermediate nodes, which led to balanced packet traffic load among the network and reduced PLR. The results in Figure 2 show that the proposed scheme achieved approximately $25 \%$ and $15 \%$ lower PLR values compared to the conventional MP-OLSR scheme at node speeds of 20 and $60 \mathrm{~m} / \mathrm{s}$, respectively. Overall, the CRAM obtained approximately $20 \%$ lower PLR compared to the MP-OLSR scheme for all node speeds.

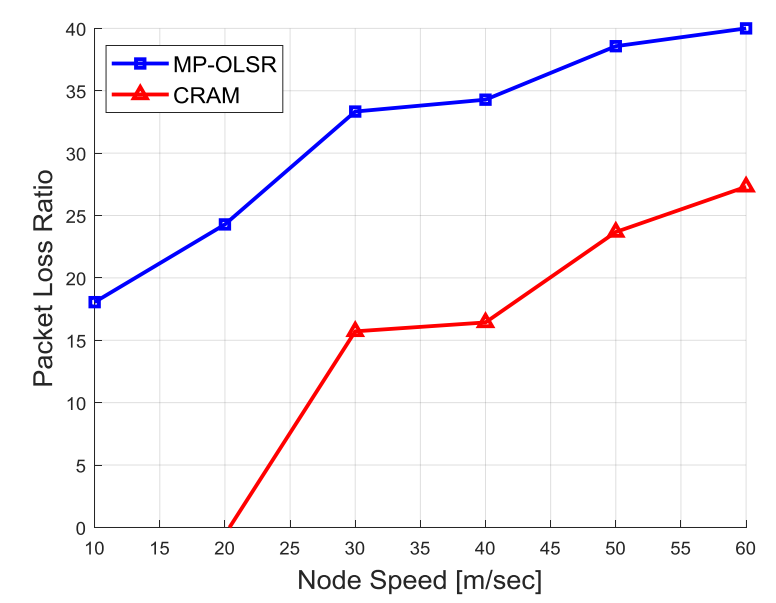

Figure 2 Packet loss ratio with different node speeds

\subsection{Energy Consumption}

The results for average energy consumption under the CRAM and MP-OLSR routing schemes are depicted in Figure 3. The proposed scheme shows less energy consumption overall, selecting paths where nodes have high battery capacity to forward data packets toward the destination node. For this reason, the scheme enhances battery capacity efficiency and balances the energy consumption of nodes in the path. Moreover, the MCDM technique provides greater weight to nodes with lower energy drain rates, enhancing energy utilization further during data transmission. It can be seen in Figure 3 that the CRAM scheme provided lesser energy consumption (approximately 57.57 and $57.87 \mathrm{mAh}$ at node speeds of 10 and $60 \mathrm{~m} / \mathrm{s}$, respectively) when compared to a conventional MP-OLSR scheme.

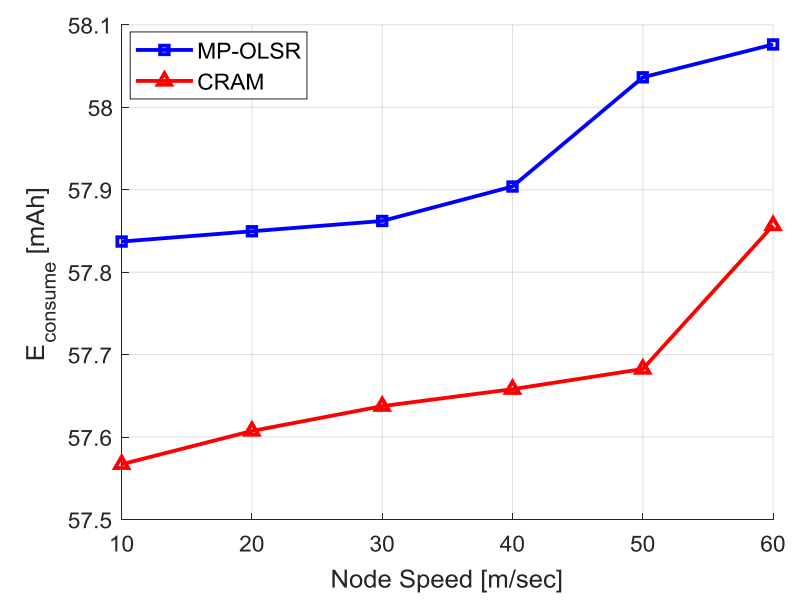

Figure 3 Energy consumption with different node speeds 


\section{CONCLUSION}

This paper has identified the traffic congestion and quickly node exhaustion constraints. To enhance network performance with load balance among the nodes and increase the network lifetime, we have presented a CRAM scheme for MANETs. The proposed approach uses CW size, which depends upon the link quality and queue length at the nodes, to provide a better chance of channel access. To reduce the energy depletion of nodes, a drain rate concept was used to provide node energy efficiency and increase network life. Under the CRAM routing scheme, optimal route selection was based on the availability status of higher energy and CW size at a node. Overall, our results proved that the proposed approach provided better performance in terms of throughput, PLR, and energy consumption when compared to the conventional MPOLSR scheme. The CRAM routing scheme is extremely applicable given a frequently changing network topology and the high speed of a wireless device, such as drone.

\section{ACKNOWLEDGEMENT}

The authors would like to acknowledge EPSRC grant EP/P028764/1 (UM IF035-2017).

\section{REFERENCES}

Atzori, L., Iera, A., Morabito, G., 2010. The Internet of Things: A Survey. Computer Networks, Volume 54(15), pp. 2787-2805

Badoi, C.-I., Prasad, N., Croitoru, V., Prasad, R., 2011. 5G based on Cognitive Radio. Wireless Personal Communications, Volume 57(3), pp. 441-464

Boushaba, A., Benabbou, A., Benabbou, R., Zahi, A., Oumsis, M., 2014. Intelligent Multipath Optimized Link State Routing Protocol for Qos and Qoe Enhancement of Video Transmission in MANETs. In: Lecture Notes in Computer Science, Springer, pp. 230245

Fatima, L., Najib, E., 2012. Mobility Support in OLSR Routing Protocol. In: Network Computing and Information Security, Springer, pp. 804-812

Hamid, B., El Mokhtar, E.-N., 2015. Performance Analysis of the Vehicular Ad-hoc Networks (VANET) Routing Protocols AODV, DSDV and OLSR. In: $5^{\text {th }}$ International Conference on Information \& Communication Technology and Accessibility (ICTA)

Hui, J.W., Vasseur, J.-P., Hong, W., 2016. Dynamic Source Route Computation to Avoid SelfInterference. Switzerland: World Intellectual Property Organization, Patentscope

Jabbar, W.A., Ismail, M., Nordin, R., 2014. Performance Evaluation of MBA-OLSR Routing Protocol for MANETs. Journal of Computer Networks and Communications, Volume 2014, pp. $1-10$

Jabbar, W.A., Ismail, M., Nordin, R., 2017. Energy and Mobility Conscious Multipath Routing Scheme for Route Stability and Load Balancing in MANETs. Simulation Modelling Practice and Theory, Volume 77, pp. 245-271

Joshi, R.D., Rege, P.P., 2012. Implementation and Analytical Modelling of Modified Optimised Link State Routing Protocol for Network Lifetime Improvement. IET Communications, Volume 6(10), pp. 1270-1277

Kabir, T., Nurain, N., Kabir, M. H., 2015. Pro-AODV (Proactive AODV): Simple Modifications to AODV for Proactively Minimizing Congestion in Vanets. In: International Conference on Networking Systems and Security (NSysS)

Lin, D., Kang, J., Squicciarini, A., Wu, Y., Gurung, S., Tonguz, O., 2017. Mozo: A Moving Zone Based Routing Protocol using Pure V2V Communication in VANETs. IEEE Transactions on Mobile Computing, Volume 16(5), pp. 1357-1370

Mumtaz, S., Huq, K.M.S., Rodriguez, J., 2014. Direct Mobile-to-Mobile Communication: Paradigm for 5G. IEEE Wireless Communications, Volume 21(5), pp. 14-23 
Peng, M., Li, Y., Zhao, Z., Wang, C., 2015. System Architecture and Key Technologies for 5G Heterogeneous Cloud Radio Access Networks. IEEE Network, Volume 29(2), pp. 6-14

Rajeswari, K., Neduncheliyan, S., 2017. Genetic Algorithm Based Fault Tolerant Clustering in Wireless Sensor Network. IET Communications, Volume 11(12), pp. 1927-1932

Sarkar, S., Datta, R., 2017. Mobility-aware Route Selection Technique for Mobile Ad Hoc Networks. IET Wireless Sensor Systems, Volume 7(3), pp. 55-64

Sarkar, S.K., Basavaraju, T.G., Puttamadappa, C., 2016. Ad Hoc Mobile Wireless Networks: Principles, Protocols, and Applications. IEEE Communications Magazine, Volume 47(5), pp. 12-14

Sarobin, V.R.M., Thomas, L.A., 2016. Improved LEACH Algorithm for Energy Efficient Clustering of Wireless Sensor Network (WSN). International Journal of Technology, Volume 7(1), pp. 50-60

Sun, Y., Sun, J., Zhao, F., Hu, Z., 2016. Delay Constraint Multipath Routing for Wireless Multimedia Ad Hoc Networks. International Journal of Communication Systems, Volume 29(1), pp. 210-225

Tilwari, V., Dimyati, K., Hindia, M.H.D., Fattouh, A., Amiri, I.S., 2019. Mobility, Residual Energy, and Link Quality Aware Multipath Routing in Manets with Q-Learning Algorithm. Applied Sciences, Volume 9(8), pp. 1-23

Tremblay, O., Dessaint, L.-A., Dekkiche, A.-I., 2007. A Generic Battery Model for the Dynamic Simulation of Hybrid Electric Vehicles. In: IEEE Vehicle Power and Propulsion Conference

Villasenor-Gonzalez, L., Ge, Y., Lament, L., 2005. HOLSR: A Hierarchical Proactive Routing Mechanism for Mobile Ad Hoc Networks. IEEE Communications Magazine, Volume 43(7), pp. 118-125

Wang, Z., Chen, Y., Li, C., 2014. PSR: A Lightweight Proactive Source Routing Protocol for Mobile Ad Hoc Networks. IEEE Transactions on Vehicular Technology, Volume 63(2), pp. $859-868$

Yi, J., Adnane, A., David, S., Parrein, B., 2011. Multipath Optimized Link State Routing for Mobile Ad Hoc Networks. Ad Hoc Networks, Volume 9(1), pp. 28-47

Yi, J., Parrein, B., 2017. Multipath Extension for the Optimized Link State Routing Protocol Version 2 (OLSRv2). Internet Engineering Task Force (IETF) 